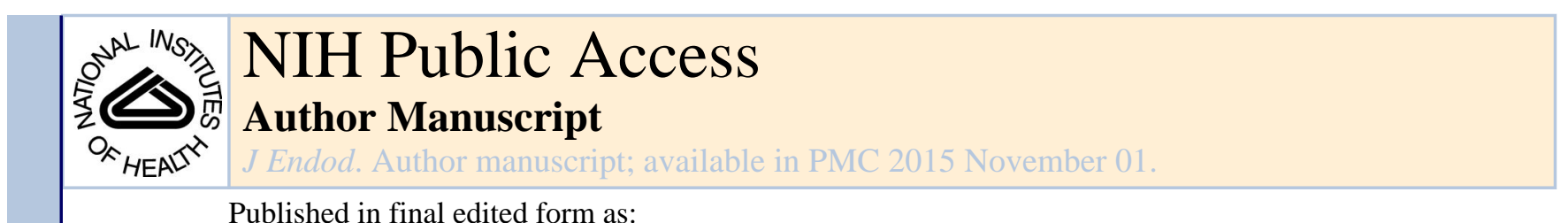

Published in final edited form as:

J Endod. 2014 November ; 40(11): 1879-1884. doi:10.1016/j.joen.2014.07.017.

\title{
Bi-Mix Antimicrobial Scaffolds for Regenerative Endodontics
}

\author{
Jadesada Palasuk, DDS, MSD ${ }^{1,2}$, Krzysztof Kamocki, DDS, MD, PhD1, Lauren \\ Hippenmeyer, BS, MS ${ }^{1}$, Jeffrey A. Platt, DDS, MS ${ }^{1}$, Kenneth J. Spolnik, DDS, MSD ${ }^{3}$, \\ Richard L. Gregory, $\mathrm{PhD}^{4}$, and Marco C. Bottino, DDS, MSc, PhD ${ }^{1, *}$
}

${ }^{1}$ Department of Restorative Dentistry/Division of Dental Biomaterials, Indiana University School of Dentistry (IUSD), Indianapolis, IN, 46202, USA 2Department of Restorative Dentistry, Faculty of Dentistry, Naresuan University, Phitsanulok, 65000, Thailand ${ }^{3}$ Department of Endodontics, IUSD, Indianapolis, IN, 46202, USA ${ }^{4}$ Department of Oral Biology, IUSD, Indianapolis, IN, 46202, USA

\begin{abstract}
Introduction-Eliminating and/or inhibiting bacterial growth within the root canal system have been shown to play a key role in the regenerative outcome. The aim of this study was to synthesize and determine in vitro both the antimicrobial effectiveness and cytocompatibility of bi-mix antibiotic-containing polydioxanone (PDS)-based polymer scaffolds.

Methods-Antibiotic-containing (metronidazole, MET and ciprofloxacin, CIP) polymer solutions (distinct antibiotic weight ratios) were spun into fibers as a potential mimic to the double antibiotic paste (DAP, a MET/CIP mixture). Fiber morphology, chemical characteristics, and tensile strength were evaluated by scanning electron microscopy (SEM), Fourier transform infrared spectroscopy (FTIR), and tensile testing, respectively. Antimicrobial efficacy was tested over time (aliquot collection) against Enterococcus faecalis (Ef), Porphyromonas gingivalis ( $\mathrm{Pg}$ ), and Fusobacterium nucleatum (Fn). Similarly, cytotoxicity was evaluated in human dental pulp stem cells (hDPSCs). Data were statistically analyzed $(\mathrm{p}<0.05)$.
\end{abstract}

Results-SEM and FTIR confirmed that electrospinning was able to produce antibioticcontaining fibers with diameter mostly in the nanoscale. Tensile strength of 1:1MET/CIP scaffolds was significantly $(\mathrm{p}<0.05)$ higher than pure PDS (control). Meanwhile, all other groups presented similar strength as the control. Aliquots obtained from antibiotic-containing scaffolds inhibited growth of $E f, P g$ and $F n$, except pure MET that did not show an inhibitory action towards $P g$ or $F n$. Antibiotic-containing aliquots promoted slight hDPSCs viability reduction, but none of them were considered to be cytotoxic.

(C) 2014 American Association of Endodontics.Published by Elsevier Inc. All rights reserved.

*Corresponding Author: Dr. Marco Cicero Bottino, Indiana University School of Dentistry, Department of Restorative Dentistry, Division of Dental Biomaterials, 1121 W. Michigan St. (DS112A), Indianapolis, IN, 46202, USA, Tel: +1-317-274-3725, fax: +1-317-278-7462. mbottino@iu.edu (M.C. Bottino).

The authors deny any conflicts of interest related to this study.

Publisher's Disclaimer: This is a PDF file of an unedited manuscript that has been accepted for publication. As a service to our customers we are providing this early version of the manuscript. The manuscript will undergo copyediting, typesetting, and review of the resulting proof before it is published in its final citable form. Please note that during the production process errors may be discovered which could affect the content, and all legal disclaimers that apply to the journal pertain. 
Conclusion-Our data suggest that the incorporation of multiple antibiotics within a nanofibrous scaffold holds great potential towards the development of a drug delivery system for regenerative endodontics.

\section{Keywords}

Electrospinning; nanofibers; scaffold; disinfection; regeneration; root canal; antibiotic; bacteria; stem cells; pulp

Traumatic injuries in addition to dental caries are the most common causes of pulp necrosis, and ultimately, if left untreated, tooth loss $(1,2)$. It is known that the incidence of pulpal necrosis in permanent teeth with incomplete apex formation (i.e., immature) poses a serious risk to fracture and thus to long-term survival, due to the thin and frequently short root (3-7). Over the past decade, root development and revascularization have been reported in the necrotic immature teeth. Concisely, this two-step regenerative procedure involves, initially, the disinfection of the root canal system with irrigation solutions and minimal instrumentation followed by placement of a medication containing a mixture of antibiotics (7-11). Generally, after 4 weeks, the medication is removed and the periapical tissue is lacerated to induce bleeding and subsequent blood clot formation (7-11). It has been assumed that the blood clot carries a variety of growth factors and acts as a natural, fibrinbased scaffold for the attachment, proliferation and differentiation of stem cells from the apical papillae $(7,10)$. Worth mentioning, eliminating and/or inhibiting bacterial growth have been shown to play a key role in the regenerative outcome $(7,9,10)$.

Knowing the limitations associated with currently advocated regenerative endodontics procedures, i.e., tooth discoloration caused by minocycline $(7,9)$, and cell toxicity $(10,12)$ associated with the use of high levels of antibiotics $(\sim 1 \mathrm{~g} / \mathrm{mL})$, our group developed, for the first time, antibiotic-containing nanofibers that can be potentially used as a drug delivery system and as a scaffold for regenerative endodontics (13). Here, distinct combinations (MET and CIP weight ratios) of antibiotic-containing polymer solutions were spun into fibers as a potential mimic to the clinically available DAP. We hypothesized that the combination MET/CIP would show (1) no antimicrobial differences against the selected bacteria, and (2) no toxicity on hDPSCs, when compared to the single antibiotic-containing scaffolds.

\section{Materials and Methods}

\section{Synthesis and Characterization of the Antibiotic-Containing Polymer Scaffolds}

Bi-mix scaffolds were synthesized via electrospinning (Figure 1A), based on the formulation of single antibiotic-containing (i.e., MET and CIP) scaffolds, the details of which have been described elsewhere (13). Scanning electron microscopy (SEM, JSM-5310LV, JEOL, Tokyo, Japan) was performed to assess fiber morphology and scaffold structure. Fiber diameter was determined from the SEM images using ImageJ (1.44i, National Institutes of Health, USA). The tensile strength of the scaffolds $\left(15 \times 3 \mathrm{~mm}^{2}, \mathrm{n}=6 /\right.$ group $)$, was evaluated by uni-axial tensile testing (expert 5601, ADMET, Norwood, MA) $(13,14)$. To confirm 
antibiotic incorporation, Fourier transform infrared spectroscopy (FTIR, Jasco 4100, Easton, MD) using attenuated total reflection (ATR) was conducted (13).

\section{Antimicrobial Testing}

Antibiotic incorporation/release was further investigated by means of an agar diffusion testing against $E f, P g$, and $F n$. Phosphate buffered saline (PBS, pH 7.4; GIBCO BRL, Grand Island, New York, USA) aliquots containing antibiotics released from the scaffolds were prepared. Briefly, rectangular-shaped scaffolds ( $\mathrm{n}=3 /$ group, $4.0 \pm 0.2 \mathrm{mg}$ ) were cut and disinfected by UV light (15 min each side) and rinsed twice with sterile PBS. Disinfected samples were placed into 24-well plates containing sterile PBS (5 mL). $500 \mu \mathrm{L}$ aliquots were withdrawn at days $1,3,7$, and 14 . An equal amount of fresh PBS was added to the wells to replace the volume removed. Aliquots were stored at $-20^{\circ} \mathrm{C}$ until used (15).

Ef (ATCC 29212) was cultured aerobically in tryptic soy broth (Difco Laboratories, Detroit, MI) for $24 \mathrm{~h}$ in $5 \% \mathrm{CO}_{2}$ at $37^{\circ} \mathrm{C}$. Meanwhile, $P g$ (ATCC 33277) and $F n$ (ATCC 25586) were cultured for $24 \mathrm{~h}$ anaerobically in Brain heart infusion (BHI) containing $5 \mathrm{~g} / \mathrm{L}$ yeast extract (Difco) with 5\% v/v Vitamin K/hemin solution (Thermo Scientific, Pittsburgh, PA) in an anaerobic GasPak jar (13). Then, $100 \mu \mathrm{L}$ of each bacterial suspension was swabbed onto blood agar plates (BAP, Fisher Scientific, Pittsburgh, PA) to create a lawn of bacteria. Each agar plate was divided into four zones and $10 \mu \mathrm{L}$ aliquots from days 1, 3, 7, and 14 were pipetted into the center of each zone. Afterwards, the BAPs were incubated according to the bacteria strain. Chlorhexidine $(0.12 \%)$ and sterile PBS served as positive and negative controls, respectively. After 2 days of incubation, either in aerobic or anaerobic conditions based on the bacteria tested, the diameters (in $\mathrm{mm}$ ) of the clear zones of growth inhibition were determined (13).

\section{Cytocompatibility}

Low glucose Dulbecco's Modified Eagle's Medium (DMEM, Gibco, Grand Island, NY) supplemented with 10\% FBS (Hyclone, Logan, UT) was used to obtain the aliquots. Similarly to the antimicrobial assay, three rectangular-shaped scaffolds per group (4.0 \pm 0.2 $\mathrm{mg}$ ) were incubated in DMEM up to 14 days (15). hDPSCs (AllCells LLC., Alameda, CA) obtained from permanent third molars were cultured in low glucose DMEM containing $10 \%$ FBS and $1 \%$ penicillin-streptomycin (Sigma) in a humidified incubator at $37^{\circ} \mathrm{C}$, with $5 \%$ $\mathrm{CO}_{2}$ (13). hDPSCs at passages 3-6 were used. Aliquots were filtered through a membrane (Millipore $^{\circledR}$ ). The positive control was a 0.3 vol \% phenol solution (13). Tests for each aliquot and time point were done in triplicate. hDPSCs were seeded at a density of $3 \times 10^{3} /$ well (100 $\mu \mathrm{L}$ cell suspension) and allowed to adhere in 96-well tissue culture microtiter plates (13). After $4 \mathrm{~h}$ of incubation the media was removed and replaced by the collected aliquots $(100 \mu \mathrm{L})$ that were adjusted to $10 \% \mathrm{FBS}$ and $1 \%$ penicillin-streptomycin and the positive control. Control (blank) wells in quadruplicate were prepared using medium without cells, and medium with cells but without the aliquot (100\% survival) (13). The microplate was then incubated in a 5\% $\mathrm{CO}_{2}$ humidified atmosphere. After 3 days, $20 \mu \mathrm{L}$ of CellTiter 96 AQueous One Solution Reagent (Promega, Madison, WI) was added to the test wells and allowed to react for $2 \mathrm{~h}$. Incorporated dye was measured by reading the absorbance at $490 \mathrm{~nm}$ in a microplate reader against blank wells $(13,16)$. 


\section{Statistical Analysis}

The fiber diameter and tensile strength of the scaffolds were analyzed using oneway ANOVA. Inhibition zone and cytocompatibility of each group was compared using an ANOVA that included a random effect to account for correlations within a specimen over time. Tukey's post-hoc test was used to count for differences among groups. The level of significance was set at $5 \%$.

\section{Results \\ Scaffolds Characterization}

Figure 1 (B-E) shows representative SEM images of the bi-mix antibiotic-containing scaffolds and the pure PDS. A micro/nanofibrous network with interconnected pores was observed for all groups. The fiber diameter of antibiotic-containing scaffolds ranged between $541.43 \pm 246.73 \mathrm{~nm}$ and $770.09 \pm 295.71 \mathrm{~nm}$, and was considerably smaller $(\mathrm{p}<0.05)$ than the pure PDS $(1179.68 \pm 449.94 \mathrm{~nm})$. The morphologic aspects of CIP and MET only scaffolds (data are not shown) are in agreement with that presented in our previous study (13). Apart from the polymer related peaks, FTIR analysis (data are not shown) confirmed the presence of the antibiotics in the scaffolds.

\section{Mechanical Strength}

Tensile strength measurements of the scaffolds (in MPa) were in an ascending order as follows: 3.45 \pm 0.51 (G4-1:3MET/CIP), 3.53 \pm 0.52 (G5-3:1MET/CIP), 4.32 \pm 0.53 (Pure PDS), $4.38 \pm 0.46$ (G1-MET only), $5.13 \pm 0.67$ (G2-CIP only), and 6.05 \pm 0.63 (G3-1:1MET/CIP). The tensile strength of G3 (1:1MET/CIP) was significantly $(\mathrm{p}<0.05)$ higher than the control (PDS). Meanwhile, all the other groups (G1, G2, G4, and G5) presented similar strengths as the control.

\section{Antimicrobial testing}

Antibiotic-containing scaffolds inhibited growth of $E f, P g$ and $F n$, except G1 (MET only) that did not show an inhibitory action towards $P g$ or $F n$ (Figure 2). Scaffolds containing higher CIP amounts (i.e., G2-CIP only, G3-1:1MET/CIP, and G4-1:3MET/CIP) had larger or at least comparable inhibition zones than those of G5 (3:1MET/CIP). The inhibition zones on $E f$ were smaller than those of $P g$ and $F n$, indicating higher antibacterial efficacy against $P g$ and $F n$.

\section{Cytocompatibility}

Figure 3 shows hDPSCs viability as a comparison within the same day antibiotic-containing aliquots. A significant reduction in cell viability was seen at day 1 for G3 (1:1MET/CIP) when compared with pure PDS (control). Furthermore, cell viability of G3 at day 1 was also significantly lower $(p<0.05)$ than that of G4 and G5. At day 14 , cell viability of G1 (MET only) was significantly higher than G2 (CIP only). No other significant differences were found. 


\section{Discussion}

One of the major goals of endodontic therapy is to eliminate microbial infection, typically a multi-species infection consisting of aerobic and anaerobic bacteria (17). The majority of bacteria inside the root canal are obligate anaerobes, thus MET is the first choice of antibiotic (17). CIP, a DNA gyrase inhibitor, is a very potent antibiotic against gramnegative pathogens but the activity is very limited against gram-positives. Consequently, it is often combined with MET in the treatment of mixed infections (18).

To the best of our knowledge, this is the first study that combined MET and CIP in a biodegradable polymer to obtain bi-mix (MET/CIP) antibiotic-containing nanofibers for application as drug delivery systems in regenerative endodontics. This study can be seen as an extension of our prior research (13) in that the use of a biocompatible bi-mix antibioticcontaining scaffold should lead to enhanced antimicrobial activity without the side-effects of staining and cell toxicity. Here, mechanical properties of these scaffolds were studied. The synthesized scaffolds demonstrated adequate mechanical properties, capable of sustaining handling and eventual placement inside root canals. Morphologically, the mean fiber diameter was in the nanoscale range and was even smaller than the pure PDS (Figure 1). This holds potential benefit because the smaller diameter creates more surface area and theoretically more drug release over an extended period of time (19-22).

Our study further demonstrated the antibacterial efficacy of the antibiotics released from the bi-mix scaffolds against $E f, P g$ and $F n$. These bacteria were chosen as the test species due to their possible microbial role in root canal infection, particularly $E f$, a common microorganism associated with refractory infection $(23,24)$. Taken together, the results from FTIR and the agar diffusion testing confirmed that antibiotics were not only incorporated into the scaffolds but the drugs were also released since inhibition of bacterial growth was seen. Generally, the higher the CIP concentration (G2-CIP only, G3-1:1MET/CIP, and G4-1:3MET/CIP), the greater the zones of inhibition. Unexpectedly, MET only (G1) did not inhibit growth of $P g$ and $F n$, thus, those results led us to reject the first null hypothesis (Figure 2). At present, and without quantitatively confirming, at each time point, the amount of drug released, for example by high performance liquid chromatography (HPLC), one plausible explanation could be that the amounts collected, based on the protocol used, might not have reached the minimal inhibitory concentrations (MIC) against $E f(>256 \mu \mathrm{g} / \mathrm{mL}$ ) (25), $P g(0.031-1.5 \mu \mathrm{g} / \mathrm{mL})(15,26,27)$, and $F n(0.242 \mu \mathrm{g} / \mathrm{mL})(28)$. It has been reported that as low as $0.001 \mathrm{mg} / \mathrm{mL}$ of double antibiotic paste (DAP) can inhibit (MIC) the growth of $E f$ and $P g(29)$. Moreover, inhibitory effects against $E f$ exhibited no significant difference between 5 wt.\% CIP and 25 wt.\% CIP (same as used in this study) containing scaffolds (13). This might suggest that the lower concentration of CIP would have shown positive results. On the other hand, MET containing scaffolds significantly inhibited growth of $P g$, disagreeing with the results observed in this study, possibly due to the different antibacterial methods used, i.e., direct contact of scaffolds with the bacteria (13), versus the released media used herein. Even though the combination of antibiotics inhibited growth of $E f, P g$ and $F n$, the inhibition might have resulted mostly from CIP, since pure MET did not inhibit the growth of sensitive bacteria $(P g$ and $F n)$. 
Apart from the necessity of evaluating the kinetics of drug release, increasing the MET concentration could also be proposed in a future investigation. The combination of MET and CIP is still needed because endodontic infections generally involve multiple species, and only three bacteria were investigated in this study. Nonetheless, it is important to highlight that the present study is a follow-up from our previous work, in which we synthesized polymer-based scaffolds containing antimicrobial drugs that are part of the double antibiotic paste (i.e., metronidazole and ciprofloxacin) at different concentrations to address their individual antimicrobial potential against endodontic-related pathogens (13). In that study, two distinct antimicrobial assays were performed to evaluate both the direct antimicrobial effect by direct contact between bacteria/scaffold, as well as on a biofilm model, in which bacteria were inoculated on the scaffolds (13). Our previous and present studies have demonstrated significant antimicrobial activity both by direct contact and by the release of the drugs on endodontic-related pathogens, respectively. Taken together, these findings have provided proof-of-concept for us to proceed with more clinically relevant investigations, such as multispecies biofilm infected dentin models.

Overall, our data revealed that the synthesized bi-mix antibiotic-containing scaffolds had slight toxicity (60\%-90\% cell viability) or non-cytotoxicity ( $>90 \%$ cell viability), therefore supporting the acceptance of our second hypothesis, where none of the groups evaluated revealed cell death below 50\% (Figure 3), according to previously established criteria (30). This may be due to the fact that we incorporated only a maximum of $25 \mathrm{wt} . \%$ (i.e., $150 \mathrm{mg}$ of drugs combined or individually) of drugs into the PDS solution and the concentration of antibiotic released may be even lower than the initial concentration, as shown previously (13).

Recent data provided in the literature strongly highlight the need of using low antibiotic concentrations prior to regenerative endodontics procedures. Together, this study demonstrated that MET- and CIP-containing scaffolds demonstrated antibacterial efficacy against $E f, P g$ and $F n$ with slight or no toxicity on hDPSCs. Therefore, within the limitations of this study, it can be concluded that using bi-mix (MET/CIP) antibiotic-containing scaffolds has great potential as an alternative medicament to DAP due to the proven antibacterial efficacy and reduced impact on cell viability.

\section{Acknowledgments}

This research was supported in part by a Research Grant from the American Association of Endodontists Foundation, by start-up funds from the IU School of Dentistry and the NIH-NIDCR (Grant \# DE023552) (all to M.C.B.).

\section{References}

1. Zero DT, Zandona AF, Vail MM, et al. Dental caries and pulpal disease. Dent Clin North Am. 2011; 55:29-46. [PubMed: 21094717]

2. Selwitz RH, Ismail AI, Pitts NB. Dental caries. Lancet. 2007; 369:51-9. [PubMed: 17208642]

3. Bose R, Nummikoski P, Hargreaves K. A retrospective evaluation of radiographic outcomes in immature teeth with necrotic root canal systems treated with regenerative endodontic procedures. $\mathbf{J}$ Endod. 2009; 35:1343-9. [PubMed: 19801227] 
4. Hargreaves KM, Geisler T, Henry M, et al. Regeneration potential of the young permanent tooth: What does the future hold? J Endod. 2008; 34:S51-6. [PubMed: 18565373]

5. Hargreaves KM, Diogenes A, Teixeira FB. Treatment options: biological basis of regenerative endodontic procedures. J Endod. 2013; 39:S30-43. [PubMed: 23439043]

6. Jung IY, Lee SJ, Hargreaves KM. Biologically based treatment of immature permanent teeth with pulpal necrosis: A case series. J Endod. 2008; 34:876-87. [PubMed: 18571000]

7. Murray PE, Garcia-Godoy F, Hargreaves KM. Regenerative endodontics: A review of current and a call for action. J Endod. 2007; 33:377-90. [PubMed: 17368324]

8. Banchs F, Trope M. Revascularization of immature permanent teeth with apical periodontitis: New treatment protocol? J Endod. 2004; 30:196-200. [PubMed: 15085044]

9. Petrino JA, Boda KK, Shambarger S, et al. Challenges in regenerative endodontics: A case series. J Endod. 2010; 36:536-41. [PubMed: 20171379]

10. Ruparel NB, Teixeira FB, Ferraz CCR, et al. Direct effect of intracanal medicaments on survival of stem cells of the apical papilla. J Endod. 2012; 38:1372-5. [PubMed: 22980180]

11. Iwaya S, Ikawa M, Kubota M. Revascularization of an immature permanent tooth with apical periodontitis and sinus tract. Dent Traumatol. 2001; 17:185-7. [PubMed: 11585146]

12. Chuensombat S, Khemaleelakul S, Chattipakorn S, et al. Cytotoxic effects and antibacterial efficacy of a 3-antibiotic combination: An in vitro study. J Endod. 2013; 39:813-9. [PubMed: 23683284]

13. Bottino MC, Kamocki K, Yassen GH, et al. Bioactive nanofibrous scaffolds for regenerative endodontics. J Dent Res. 2013; 92:963-9. [PubMed: 24056225]

14. Bottino M, Yassen G, Platt J, et al. A novel three-dimensional scaffold for regenerative endodontics:materials and biological characterizations. J Tissue Eng Regen Med. 201310.1002/ term. 1712

15. Reise M, Wyrwa R, Muller U, et al. Release of metronidazole from electrospun poly(L-lactide-coD/L-lactide) fibers for local periodontitis treatment. Dent Mater. 2012; 28:179-88. [PubMed: 22226009]

16. Bottino MC, Coelho PG, Henriques VAR, et al. Processing, characterization, and in vitro/in vivo evaluations of powder metallurgy processed Ti-13Nb-13Zr alloys. J Biomed Mater Res A. 2009; 88:689-96. [PubMed: 18335528]

17. Vijayaraghavan R, Mathian VM, Sundaram AM, et al. Triple antibiotic paste in root canal therapy. J Pharm Bioallied Sci. 2012; 4:S230-3. [PubMed: 23066258]

18. Windley W 3rd, Teixeira F, Levin L, et al. Disinfection of immature teeth with a triple antibiotic paste. J Endod. 2005; 31:439-43. [PubMed: 15917683]

19. Cui WG, Li XH, Zhu XL, et al. Investigation of drug release and matrix degradation of electrospun poly(DL-lactide) fibers with paracetanol inoculation. Biomacromolecules. 2006; 7:1623-9. [PubMed: 16677047]

20. Kenawy ER, Bowlin GL, Mansfield K, et al. Release of tetracycline hydrochloride from electrospun poly(ethylene-co-vinylacetate), poly(lactic acid), and a blend. J Control Release. 2002; 81:57-64. [PubMed: 11992678]

21. Kenawy ER, Abdel-Hay FI, El-Newehy MH, et al. Controlled release of ketoprofen from electrospun poly(vinyl alcohol) nanofibers. Mat Sci Eng A-Struct. 2007; 459:390-6.

22. Kenawy ER, Abdel-Hay FI, El-Newehy MH, et al. Processing of polymer nanofibers through electrospinning as drug delivery systems. Mater Chem Phys. 2009; 113:296-302.

23. Madhubala MM, Srinivasan N, Ahamed S. Comparative evaluation of propolis and triantibiotic mixture as an intracanal medicament against Enterococcus faecalis. J Endod. 2011; 37:1287-9. [PubMed: 21846550]

24. Pallotta RC, Ribeiro MS, de Lima Machado ME. Determination of the minimum inhibitory concentration of four medicaments used as intracanal medication. Aust Endod J. 2007; 33:107-11. [PubMed: 18076578]

25. Dahlen G, Samuelsson W, Molander A, et al. Identification and antimicrobial susceptibility of enterococci isolated from the root canal. Oral Microbiol Immunol. 2000; 15(5):309-12. [PubMed: 11154422] 
26. Kulik EM, Lenkeit K, Chenaux S, et al. Antimicrobial susceptibility of periodontopathogenic bacteria. J Antimicrob Chemother. 2008; 61:1087-91. [PubMed: 18326855]

27. Eick S, Seltmann T, Pfister W. Efficacy of antibiotics to strains of periodontopathogenic bacteria within a single species biofilm - an in vitro study. J Clin Periodontol. 2004; 31:376-83. [PubMed: 15086620]

28. Poulet PP, Duffaut D, Lodter JP. Metronidazole susceptibility testing of anaerobic bacteria associated with periodontal disease. J Clin Periodontol. 1999; 26:261-3. [PubMed: 10223399]

29. Sabrah AHA, Yassen GH, Gregory RL. Effectiveness of Antibiotic Medicaments against Biofilm Formation of Enterococcus faecalis and Porphyromonas gingivalis. J Endod. 2013; 39:1385-9. [PubMed: 24139259]

30. Dahl JE, Frangou-Polyzois MJ, Polyzois GL. In vitro biocompatibility of denture relining materials. Gerodontology. 2006; 23(1):17-22. [PubMed: 16433637] 


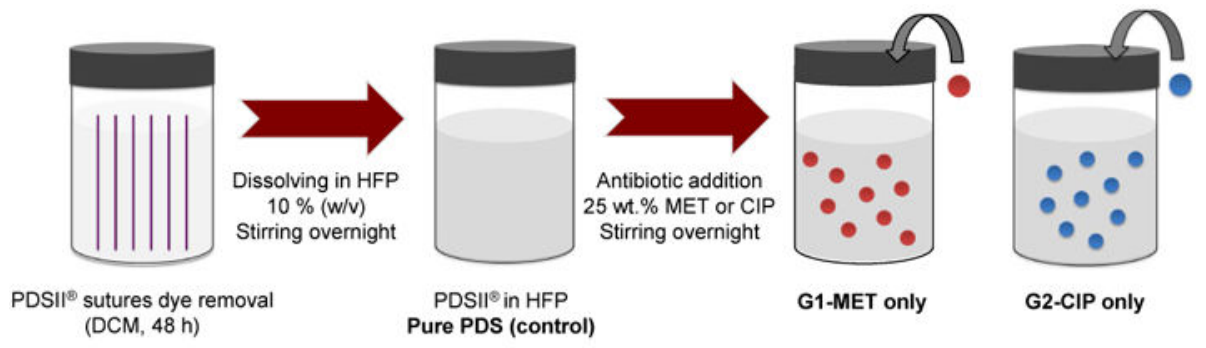

Bi-Mix formulations (by weight)
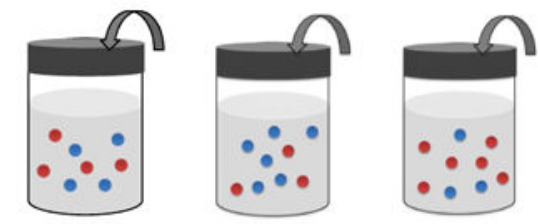

G3-1:1MET/CIP

G4-1:3MET/CIP

G5-3:1MET/CIP

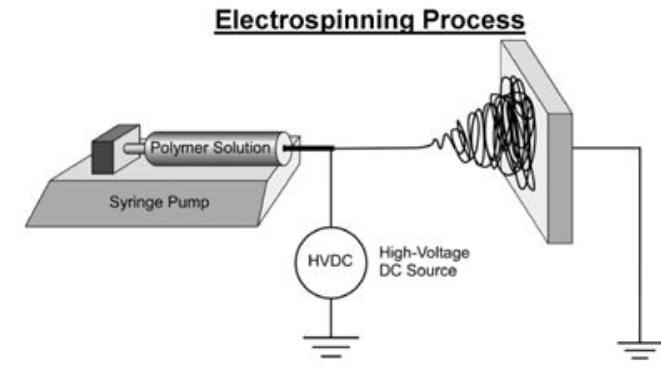

$1 \mathrm{~A}$.
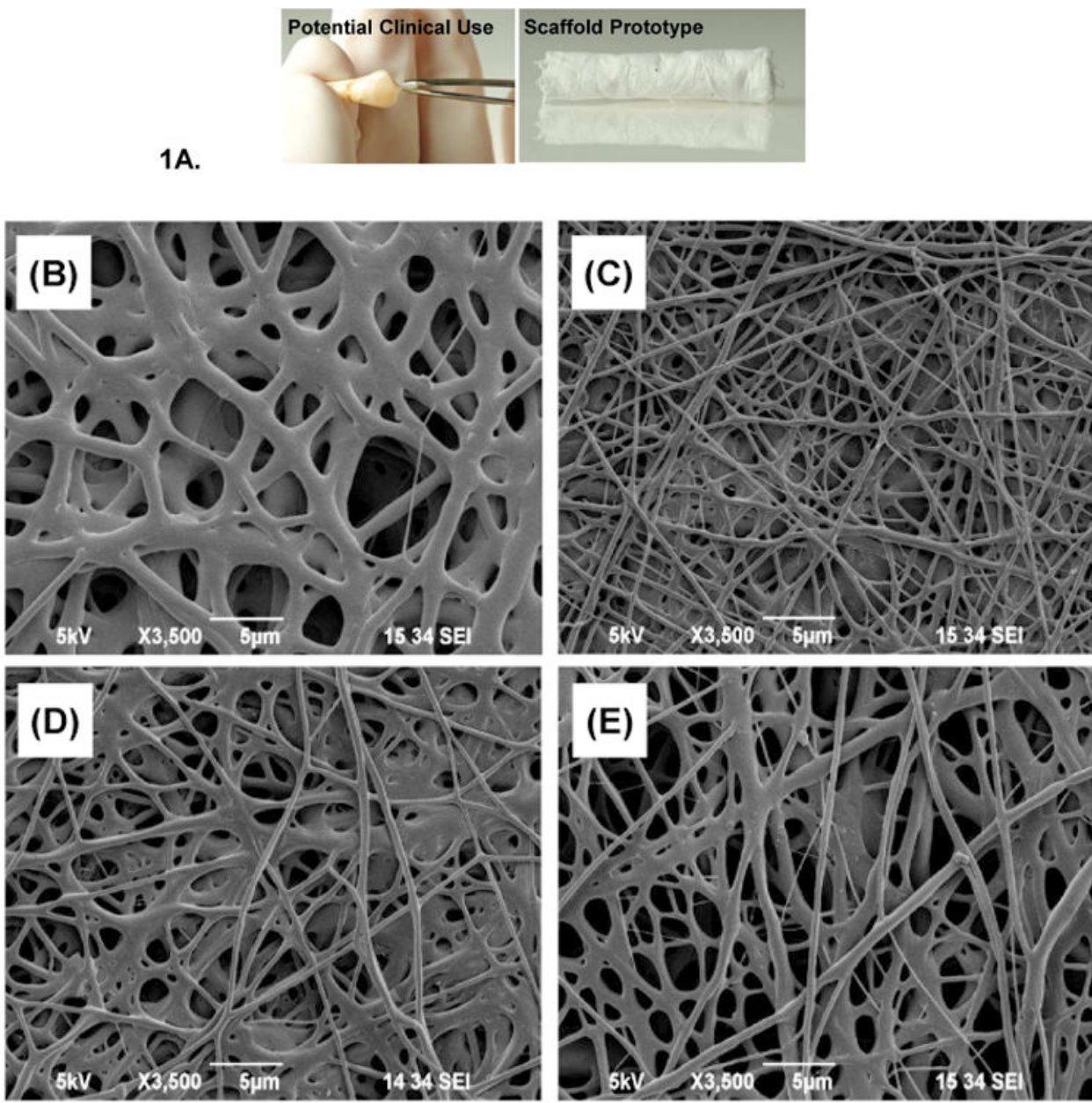

Figure 1.

(A) Polydioxanone suture filaments (PDS II ${ }^{\circledR}$, Ethicon, Somerville, NJ) were first undyed using dichloromethane (Sigma-Aldrich, St. Louis, MO) and then dissolved in 1,1,1,3,3,3- 
hexafluoro-2-propanol (HFP, Sigma-Aldrich) at $10 \mathrm{wt} . \%$ under stirring conditions. MET (Sigma-Aldrich) and CIP (Sigma-Aldrich) containing solutions were prepared by adding MET (G1) and CIP (G2) into the PDS solution at a $25 \mathrm{wt} . \%$ relative to the total PDS weight (i.e., $150 \mathrm{mg}$ of the antibiotic) and mixed together under vigorous stirring. Different ratios (by weight) of the two antibiotics were added to PDS solutions and mixed under vigorous stirring to obtain the following antibiotic-containing solutions: G3-1:1MET/CIP (75 mg of each antibiotic), G4-1:3MET/CIP (37.5 mg/112.5 mg), and G5-3:1MET/CIP (112.5 mg/37.5 $\mathrm{mg}$ ). The antibiotic-containing and pure PDS (control) solutions were electrospun under optimized parameters. The electrospun scaffolds (hereafter referred to as mats) were desiccated under vacuum at ambient conditions for $48 \mathrm{~h}$ (13). (B-E) Representative SEM micrographs of antibiotic-containing mats at 3,500x: (B) G6-Pure PDS; (C) G3-1:1MET/ CIP; (D) G4-1:3MET/CIP, and (E) G5-3:1MET/CIP. 
A

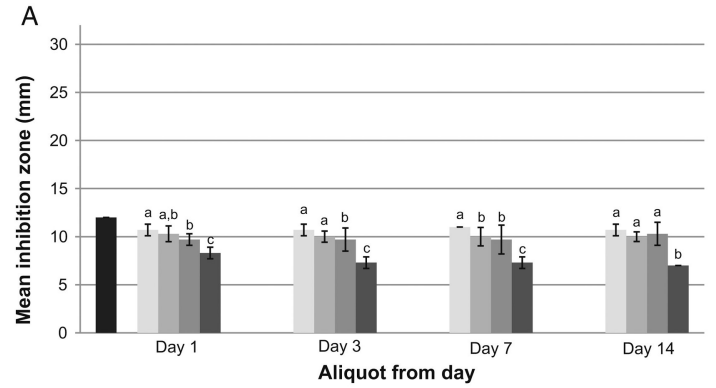

- $0.12 \% \mathrm{CHX}=\mathrm{G} 2-\mathrm{CIP}$ only $=\mathrm{G} 3-1: 1 \mathrm{MET} / \mathrm{CIP}=\mathrm{G} 4-1: 3 \mathrm{MET} / \mathrm{CIP}=\mathrm{G} 5-3: 1 \mathrm{MET} / \mathrm{CIP}$

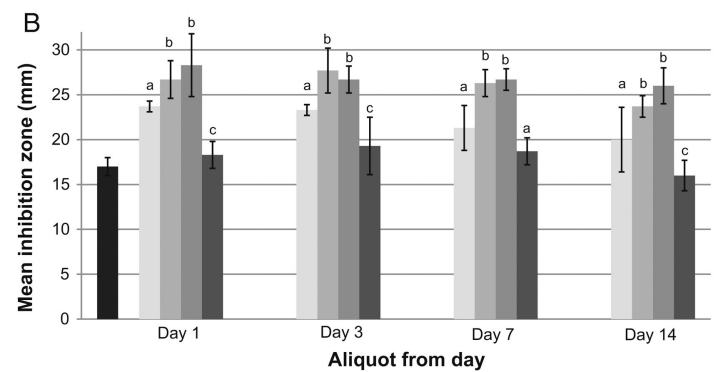

- 0.12\% CHX $\approx$ G2-CIP only $=$ G3-1:1MET/CIP $=$ G4-1:3MET/CIP $=$ G5-3:1MET/CIP

C

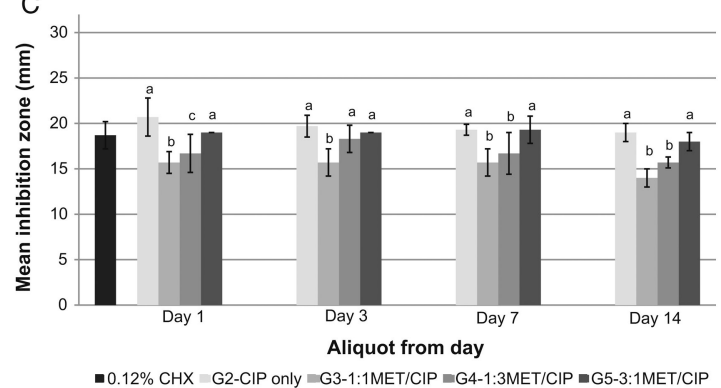

Figure 2.

Results from agar diffusion assays are represented as mean inhibition zone (in $\mathrm{mm}$ ) against the different bacteria tested: (A) E. faecalis; (B) P. gingivalis; and (C) F. nucleatum. Same letters indicate insignificant difference compared to the results of the same day of aliquots. Aliquots from PBS (negative control), Pure PDS (control), and G1 (MET only) showed no inhibition zone on the three tested bacteria and are not shown. 


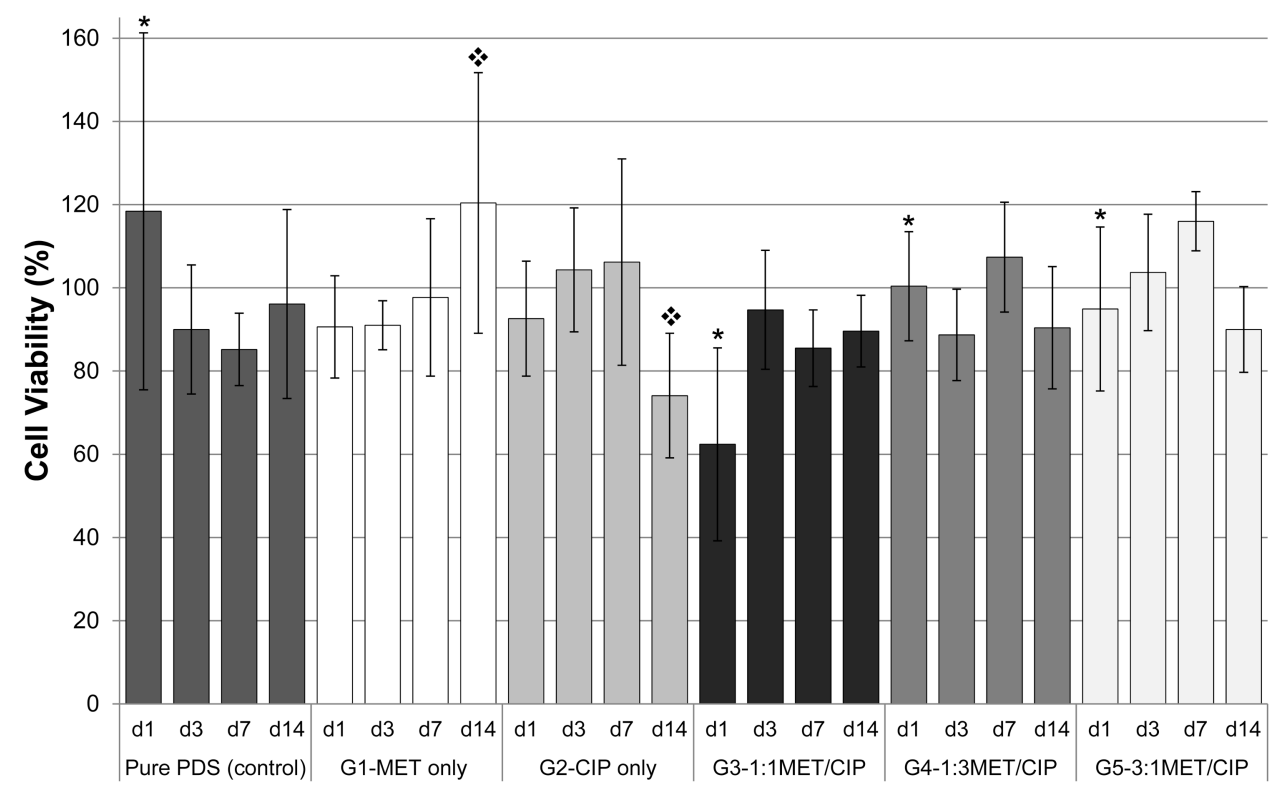

Figure 3.

Cytotoxicity assay measured viability (\%) of human dental pulp stem cells (hDPSCs) in response to aliquots at day 1, 3, 7 and 14 from electrospun mats. Statistical analyses were compared with the same day results. *At day 1 , cell viability of G3-1:1MET/CIP was significantly lower than that of Pure PDS, G4-1:3MET/CIP, and G5-3:1MET/CIP. * At day 14 , cell viability of G1-MET only was significantly higher than that of G2-CIP only. No other significant differences were found among the other groups. 